\title{
Psicologia Positiva e os Instrumentos de Avaliação no Contexto Brasileiro
}

\section{Positive Psychology and Evaluating Instruments Used in Brazil}

\author{
Fabio Scorsolini-Comin ${ }^{a} \&$ Manoel Antonio dos Santos, ${ }^{*} b$ \\ ${ }^{a}$ Universidade Federal do Triangulo Mineiro, Uberaba, Brasil \& ${ }^{b}$ Universidade de São Paulo, Ribeirão Preto, Brasil
}

\begin{abstract}
Resumo
O objetivo é apresentar uma revisão integrativa da literatura científica acerca dos instrumentos de avaliação existentes na Psicologia Positiva, no contexto brasileiro. Pelas bases LILACS e SciELO (1970-2008), a partir de critérios de inclusão/exclusão, foram recuperados e analisados seis trabalhos. Os estudos que abordam os instrumentos de mensuração existentes são relacionados ao bem-estar subjetivo e à satisfação. Entre as principais contribuições do referencial, destaca-se a construção de instrumentos de avaliação e modelos de intervenção. As principais escalas mencionadas são norte-americanas, havendo apenas uma brasileira. Constatou-se a necessidade de se firmar a produção de instrumentos adaptados e validados para o contexto brasileiro, além de novos estudos que investiguem os instrumentos internacionais, seus pressupostos, validação e aplicação em diversos contextos culturais.

Palavras-chave: Psicologia Positiva; Felicidade; Avaliação; Escalas; Revisão.
\end{abstract}

\begin{abstract}
The objective of this study is to present an integrative review of the scientific literature about concepts, methods and evaluating instruments regarding Positive Psychology. Six papers from LILACS and SCIELO databases (1970-2008) based on inclusion/exclusion criteria were analyzed. The studies broached tools of measurement related to subjective welfare and satisfaction. Among the major contributions of this frame of reference, we point out the construction of estimation tools and intervention models. The foremost scales are North-American, in fact, there is just one Brazilian scale. Thus, it was noticed the necessity of developing adapted and valid instruments for the Brazilian context. Besides, new studies that investigate international instruments, their prerequisites, validation and applications in different cultural contexts should be done.

Keywords: Positive Psychology; Happiness; Evaluation; Scales; Review.
\end{abstract}

Durante muito tempo, a Psicologia deu ênfase às questões relacionadas à doença, não se atendo às discussões sobre a saúde e o bem-estar (Diener, 1984). É nessa lacuna de investigações que se situa a Psicologia Positiva. Nesta corrente, a felicidade pode ser definida como a predominância da frequência de ocorrência de experiências emocionais positivas sobre as negativas, ou seja, afetos positivos mais frequentes do que os negativos. Para Sheldon e King (2001), a Psicologia Positiva é o estudo científico das forças e virtudes próprias do indivíduo. Para Seligman (2004), trata-se do estudo de sentimentos, emoções, instituições e comportamentos positivos que têm como objetivo final a felicidade humana.

\footnotetext{
Endereço para correspondência: Professor Fabio Scorsolini-Comin, Departamento de Psicologia do Desenvolvimento, da Educação e do Trabalho, Universidade Federal do Triângulo Mineiro, Avenida Getúlio Guaritá, 159, $3^{\circ}$ andar, Abadia, Uberaba, MG, CEP 38025-440. E-mail: masantos@ffclrp.usp.bre scorsolini_usp@yahoo.com.br Os autores agradecem à Fundação de Amparo à Pesquisa do Estado de São Paulo (FAPESP) pelo financiamento do trabalho (proc. 2007/52584-5).
}

De acordo com o trabalho de resgate histórico promovido por Graziano (2005), a Psicologia Positiva teve início com os estudos de Terman, Jung e Watson na década de 1930 sobre superdotados, sucesso no casamento e paternidade. No entanto, o estudo sobre a felicidade e sobre as emoções positivas se iniciou por volta da década de 1950, quando a Organização das Nações Unidas passou a ter interesse ativo na mensuração dos níveis de vida de várias comunidades mundiais, o que hoje se designa por expressões correlatas, tais como bem-estar, condições de vida ou qualidade de vida (Pereira, 1997).

Todavia, cientificamente, o movimento batizado de Psicologia Positiva surgiu nos Estados Unidos em 1998, a partir da iniciativa de Martin Seligman que, com a colaboração de outros pesquisadores, começou a desenvolver pesquisas quantitativas visando promover uma mudança no foco atual da Psicologia ou de abordagens atuais (Graziano, 2005). Essa perspectiva propõe, basicamente, a modificação do foco da Psicologia de uma reparação dos aspectos ruins da vida para a construção 
de qualidades positivas (Caprara \& Steca, 2006; Rijavec, Brdar, \& Miljkovic, 2006; Seligman, 2004).

Segundo Seligman (2004), a Psicologia Positiva se sustenta sobre três pilares principais, a saber: o estudo da emoção positiva; o estudo dos traços ou qualidades positivas, principalmente forças e virtudes, incluindo habilidades como inteligência e capacidade atlética; e, por fim, o estudo das chamadas instituições positivas, como a democracia, a família e a liberdade - que dão suporte às virtudes que, por sua vez, oferecem apoio às emoções positivas.

Em relação às boas e más qualidades, Graziano (2005) afirma que, partindo da ideia de que seriam positivas as emoções que favorecem a interação e negativas aquelas que a prejudicam, a Psicologia Positiva define que as boas qualidades são as características humanas que favorecem as emoções positivas e o comportamento de interação, de forma que as más qualidades seriam o oposto, ou seja, as características humanas que favorecem as emoções negativas, bem como o comportamento que prejudica a interação.

A Psicologia Positiva pretende se debruçar sobre as experiências positivas (como emoções positivas, felicidade, esperança e alegria), características positivas individuais (como caráter, forças e virtudes), e instituições positivas (como organizações baseadas no sucesso e potencial humano, sejam locais de trabalho, escolas, famílias, hospitais, comunidades ou sociedades) (Larrauri, 2006; Marujo, Neto, Caetano, \& Rivero, 2007; Park \& Peterson, 2007; Seligman, 2004).

Essa corrente resgata o caráter preventivo que, há muito, fora abandonado por uma Psicologia exclusivamente focalizada na doença. Por meio do estudo das características humanas positivas, a ciência aprenderá a prevenir doenças físicas e mentais e os psicólogos, por sua vez, aprenderão a desenvolver qualidades que ajudem indivíduos e comunidades a bem mais do que resistir ou sobreviver, mas efetivamente a florescer (Csikszentmihalyi, 2006; Passareli \& Silva, 2007; Seligman, 2000; Serbena \& Raffaelli, 2003).

De acordo com essa nova visão, o conhecimento das forças e virtudes poderia propiciar o florescimento (flourishing) das potencialidades das pessoas, comunidades e instituições. Segundo Paludo e Koller (2007), florescimento tem sido um termo bastante utilizado na Psicologia Positiva. Trata-se de uma condição que permite o desenvolvimento pleno, saudável e positivo dos aspectos psicológicos, biológicos e sociais dos seres humanos.

A Psicologia Positiva, enquanto área do saber psicológico, não promove uma novidade, não cria uma nova realidade, mas é um exercício teórico e metodológico no sentido de mudar a visão que se lança aos fenômenos investigados pela Psicologia, em uma proposta que evidencia os aspectos positivos e salutares do desenvolvimento, nos contornos de uma compreensão que prioriza a prevenção (Albuquerque \& Tróccoli, 2004; Diener, 1984; Seligman, 2004; Seligman, Steen, Park, \& Peterson, 2006) e o florescimento de aspectos positivos que possam ter valor adaptativo (Graziano, 2005).

Enquanto corrente surgida nos Estados Unidos, sua repercussão no cenário latinoamericano ainda é tímida quando comparada aos países europeus (Delle Fave, 2006; Marujo et al., 2007; Scorsolini-Comin \& Santos, 2009), mas tem encontrado no Brasil um campo fértil para a produção de saber científico de impacto (Albuquerque \& Tróccoli, 2004; Graziano, 2005; Paludo \& Koller, 2007; Paschoal \& Tamayo, 2008; Passareli \& Silva, 2007; Scorsolini-Comin \& Santos, 2010).

No estudo de Calvetti, Muller e Nunes (2007), empreendeu-se uma revisão na base LILACS, no período de 2000 a 2006. Segundo as autoras, foram encontrados poucos estudos na perspectiva da Psicologia Positiva, bem como de conceitos correlatos, como resiliência, religiosidade, espiritualidade, otimismo e esperança. Até o ano da publicação do trabalho (2006), ainda não havia um grupo de trabalho na Associação Nacional de Pesquisa e Pós-Graduação em Psicologia (ANPEPP) dedicado à Psicologia Positiva, embora possa se destacar um encontro realizado em Florianópolis e a publicação do livro Resiliência e Psicologia Positiva (Dell'Aglio, Koller, \& Yunes, 2006). Para Calvetti et al. (2007), observava-se, à época, um movimento que iniciava a consolidação dessa área. Essa constatação permite que se indague como esta realidade se apresenta atualmente.

Nos limites desse campo de investigação há uma série de trabalhos que destacam escalas e instrumentos utilizados para mensurar a felicidade das pessoas - ou, em outras palavras, as noções de bem-estar subjetivo, afetos positivos e satisfação com a vida, por exemplo. Esses instrumentos, em sua franca maioria, foram desenvolvidos e validados no âmbito internacional. Pensando nos estudos da Psicologia Positiva no contexto brasileiro e tendo em vista o possível amadurecimento dessa perspectiva, quais seriam os instrumentos mais utilizados pelos autores nacionais? Seriam adaptações daqueles utilizados nos países centrais, como Estados Unidos e Europa? Ou seriam instrumentos produzidos especialmente para o cenário brasileiro? Quais os pressupostos desses instrumentos e como eles repercutem na construção do saber da Psicologia Positiva, notadamente no Brasil?

Considerando essa questão, este estudo pretende contribuir para fortalecer essa discussão a partir de uma revisão integrativa da literatura científica acerca de seus instrumentos de mensuração utilizados. Busca-se evidenciar o perfil dos artigos publicados no contexto brasileiro, de modo a possibilitar um maior direcionamento das pesquisas sobre esse referencial teórico e discutir as tendências, lacunas e perspectivas de produção científica na área da avaliação em Psicologia Positiva. 


\section{Método}

\section{Tipo de Estudo}

Trata-se de uma revisão integrativa. De acordo com Beyea e Nicoll (1998), tal revisão sumariza pesquisas passadas e tira conclusões globais de um corpo de literatura de um tópico em particular (no caso, a mensuração na Psicologia Positiva), contribuindo para discussões sobre métodos e resultados de pesquisa, assim como reflexões sobre a realização de futuras pesquisas. Seguindo os procedimentos preconizados por Ganong (1987), como o estabelecimento de critérios para inclusão e exclusão dos estudos e análise crítica dos resultados, observa-se que, embora os métodos para a condução de revisões integrativas variem, existem padrões a serem seguidos. É necessário, portanto, seguir padrões de rigor e clareza na revisão e crítica das publicações selecionadas, de forma que o leitor possa identificar as características salientes dos estudos revisados.

$\mathrm{Na}$ operacionalização desta revisão utilizamos as seguintes etapas: seleção da questão temática, estabelecimento dos critérios para a seleção da amostra, análise e interpretação dos resultados e apresentação da revisão.

\section{Procedimentos}

Os unitermos de busca utilizados foram: Psicologia Positiva (Positive Psychology) e bem-estar subjetivo (subjective well-being). O bem-estar subjetivo foi utilizado como termo de busca, haja vista que, internacionalmente, essa noção está fortemente associada à Psicologia Positiva e à mensuração. Uma vez concluído o levantamento das publicações, os resumos foram lidos e analisados segundo os critérios de inclusão/exclusão estabelecidos. Os trabalhos selecionados foram resgatados e analisados na íntegra.

Bases de Dados Consultadas. Visando assegurar uma ampla abrangência desta revisão no contexto brasileiro, foram consultadas as seguintes bases de dados: LILACS e SciELO.

Critérios de Inclusão e Exclusão dos Trabalhos. Foram excluídos trabalhos como artigos não indexados, teses, dissertações, resenhas, livros e capítulos de livros. Essa exclusão deveu-se ao fato de que tais publicações não foram submetidas a um processo às cegas de avaliação de pares, que é um critério amplamente aceito de garantia de qualidade do conhecimento produzido (Scorsolini-Comin \& Amorim, 2008). Desse modo, optou-se pela seleção de artigos publicados em revistas indexadas apenas. Obviamente, a ampliação das fontes nos levaria a outros resultados, mas poderiam, em certa medida, comprometer a avaliação da qualidade dos mesmos. Como se trata de uma área ainda em formação, vem se notando que alguns estudos ainda não primam por uma adequada sistematização, estando, muitas vezes, presos a um mero mapeamento da perspectiva que, em- bora seja necessário, pouco contribui para o avanço teórico-metodológico. Foram excluídos, ainda, trabalhos cuja temática mostrou-se distante do tema, como estudos relacionados exclusivamente à área médica (sem qualquer relação com a Psicologia Positiva) e à Psicologia Experimental (o unitermo Psicologia Positiva permitiu resgatar diversos trabalhos acerca do reforçamento positivo, por exemplo, que não guardavam qualquer relação com a área investigada nesta revisão).

O levantamento compreendeu o período de 1970 a 2008. Tal abrangência objetivou traçar um perfil das publicações no período, ainda que caiba aqui a ressalva de que essa área (Psicologia Positiva) desenvolveu-se, fundamentalmente, a partir da década de 1990 (Seligman, 2004). Como critérios de inclusão, destacamos artigos empíricos, teóricos e de revisão acerca do tema (mensuração na Psicologia Positiva), que se referiam ao contexto brasileiro ou que tivessem sido produzidos no país.

\section{Resultados}

Nas bases bibliográficas selecionadas foram encontrados 246 trabalhos (resumos) pelos unitermos de busca. Entre os trabalhos excluídos já a partir da leitura dos resumos, a maioria se referia a estudos de caso de patologias específicas como câncer, diabetes mellitus e outras condições, além de investigações sobre prevalência e tratamentos de doenças, sem qualquer ligação com a Psicologia Positiva. Outro importante filão de produção excluída está relacionado à Psicologia Experimental, dentro da perspectiva da Psicologia Comportamental, ou seja, trabalhos que não abordavam a Psicologia Positiva, mas a lei do reforço e outros construtos não ligados à área investigada. Outro eixo de destaque encontrado foram os trabalhos acerca do HIV/Aids e da vivência da soropositividade, sem relação com a perspectiva da Psicologia Positiva.

Outros eixos excluídos foram: Psicologia Clínica, educação, antropologia/filosofia/religião, testes psicológicos, habilidades sociais, inclusão, Psicologia Ambiental, idosos, saúde no trabalho, arte, homossexualidade, cooperativismo, educação musical, violência, adolescência e ecologia do desenvolvimento. Deve-se destacar que tais estudos não foram excluídos por suas temáticas e sim por não estabelecerem qualquer nexo com a Psicologia Positiva. Do mesmo modo, estudos que trouxeram a mera menção à Psicologia Positiva também foram excluídos, uma vez que não poderiam contribuir para a discussão objetivada pela presente investigação.

A partir da utilização dos critérios de inclusão e exclusão adotados, chegou-se a um total de apenas seis artigos indexados, produzidos no contexto brasileiro, que abordavam instrumentos de mensuração na Psicologia Positiva. Essa amostra reduzida pode ser relacionada tanto ao amadurecimento de estudos na perspectiva da Psicologia ao longo dos anos da década de 1990 e da década em curso, quanto ao grau de especificidade da busca. A 
avaliação ocupa um papel de destaque na ciência psicológica e, assim como a Psicologia Positiva, vem sendo paulatinamente amadurecida pelas recentes pesquisas, a avaliação neste referencial ainda não é frequentemente empreendida de modo sistemático.
Esses estudos foram recuperados e analisados na íntegra. Os estudos-alvo desta revisão foram agrupados na Tabela 1.

Tabela 1

Instrumentos de Mensuração Mencionados elou Investigados nos Estudos Selecionados

\begin{tabular}{|c|c|c|}
\hline Autores & Ano de publicação & Instrumentos de mensuração mencionados/investigados \\
\hline Albuquerque \& Tróccoli & 2004 & Escala de Bem-estar Subjetivo (EBES); PANAS; SWBS; SWLS \\
\hline Dela Coleta \& Dela Coleta & 2006 & $\begin{array}{l}\text { Não há menção específica aos instrumentos. Bateria de escalas } \\
\text { adaptadas daquelas apresentadas por Diener, Diener e Diener } \\
\text { (1995), Diener e Suh (2000) e Seligman (2004) }\end{array}$ \\
\hline Resende, Bones, \& Souza & 2006 & Escala do tipo item único para satisfação com a vida \\
\hline Paludo \& Koller & 2007 & $\begin{array}{l}\text { Não há menção específica aos instrumentos, mas destaca } \\
\text { a importância da mensuração na Psicologia Positiva }\end{array}$ \\
\hline Ferraz, Tavares, \& Zilberman & 2007 & $\begin{array}{l}\text { PANAS-X; SWLS; OHI; SHS; DHS; DHS-Brief; } \\
\text { Life Satisfaction Inventory: Form A }\end{array}$ \\
\hline Paschoal \& Tamayo & 2007 & PANAS \\
\hline
\end{tabular}

A maioria dos estudos encontrados se referia à Psicologia como área mais ampla ou a aspectos positivos de determinada área, como a questão do reforçamento na terapia comportamental ou na Psicologia Experimental. Assim, foram escassos os trabalhos que efetivamente abordavam a Psicologia Positiva enquanto área de estudo, os quais foram integralmente selecionados para análise e discussão. Ressalta-se, ainda, que esse campo de investigação, embora seja atual e venha sendo discutido pela Psicologia de modo crescente, notadamente a partir de 1998, ainda conta com poucos estudos de revisão ou trabalhos empíricos que adotem tal referencial. Em alguns trabalhos, os autores apenas mencionam a assunção da Psicologia Positiva sem, no entanto, utilizá-la efetivamente para sustentar suas conclusões ou desenvolver conceitos abordados nesta área. Tal colocação deve ser entendida como um convite para novas pesquisas que explorem este referencial, notadamente investigações sobre os instrumentos de mensuração utilizados no contexto nacional e latinomaericano.

Em relação ao ano de publicação dos trabalhos selecionados, observa-se que todos são da década em curso, ou seja, todos os trabalhos selecionados foram publicados a partir de 2004, o que mostra que, embora as discussões relativas a essa perspectiva sejam anteriores, como observado anteriormente, o pico de produção científica se deu na presente década, com acentuado crescimento nos últimos anos (2007 e 2008).

No que se refere ao perfil dos trabalhos selecionados e recuperados, todos apresentam uma breve contextualização da área da Psicologia Positiva. Esse movimento é compreendido como uma aproximação das pesquisas em relação à comunicação científica, uma vez que esse campo de investigação é relativamente recente. Encontraram-se importantes artigos de revisão histórica, destacando a origem e o surgimento da Psicologia Positiva (Ferraz et al., 2007; Paludo \& Koller, 2007).

Dentre os estudos excluídos por não trabalharem com os instrumentos de mensuração, destacam-se pesquisas que investigam a Psicologia Positiva e as suas implicações para a ciência psicológica (Contreras \& Esguerra, 2006; Serbena \& Raffaelli, 2003; Yunes, 2003). Outros trabalhos destacaram os conceitos existentes na Psicologia Positiva, como o bem-estar subjetivo (Guedea et al., 2006; Neri, 1998; Passareli \& Silva, 2007), bem como sua correlação com as noções de resiliência (Yunes, 2003), self (Neri, 2002), estratégias de enfrentamento, apoio social e variáveis sociodemográficas (Guedea et al., 2006). Em relação às populações estudadas nos trabalhos empíricos, destacam-se os idosos (Guedea et al., 2006; Neri, 2002).

A seguir, dado o objetivo deste estudo, serão apresentados e discutidos os trabalhos relativos aos instrumentos de mensuração e de avaliação da Psicologia Positiva, publicados e/ou desenvolvidos no contexto brasileiro de produção.

\section{Análise e Discussão dos Trabalhos}

\section{Psicologia Positiva: Um Campo \\ Em Florescimento no Brasil}

Em um primeiro estudo resgatado, Paludo e Koller (2007) descrevem que a Psicologia Positiva está em pleno processo de expansão dentro da ciência psicológica, o que possibilita uma reavaliação das potencialidades e virtudes humanas por meio do estudo das condições e 
processos que contribuem para a prosperidade. De acordo com essa nova visão, o conhecimento das forças e virtudes poderia propiciar o "florescimento (flourishing) das pessoas, comunidades e instituições" (Paludo \& Koller, 2007, p. 10). Segundo as autoras, florescimento tem sido um termo bastante utilizado na Psicologia Positiva. Trata-se de uma condição que permite o desenvolvimento pleno, saudável e positivo dos aspectos psicológicos, biológicos e sociais dos seres humanos. O florescimento significa um estado no qual os indivíduos sentem uma emoção positiva pela vida, apresentam um ótimo funcionamento emocional e social e não possuem problemas relacionados à saúde mental.

Segundo a abrangente revisão de Paludo e Koller (2007), entre as principais contribuições da Psicologia Positiva destacam-se: a construção de instrumentos de avaliação, modelos de intervenção e aplicação no curso desenvolvimental. Essa proposta teórica pretende criar métodos preventivos por meio do conhecimento dos fatores protetivos, aprimorar técnicas de avaliação psicológica para identificação das virtudes e dos aspectos positivos e ampliar o escopo de estudo das Ciências Sociais e Humanas. No entanto, o referido artigo não menciona quais seriam esses instrumentos de mensuração, nem quais critérios psicométricos ou conceituais deveriam nortear sua construção. Este foi o único estudo selecionado que não aborda especificamente os instrumentos existentes na Psicologia Positiva, mas que destaca a relevância dessas avaliações nesse campo em desenvolvimento. Tais instrumentos, escalas ou baterias de testes serão destacados a seguir.

\section{A Mensuração na Perspectiva da Psicologia Positiva}

No que concerne aos instrumentos de avaliação do bem-estar subjetivo (BES) na perspectiva da Psicologia Positiva, destaca-se uma bateria desenvolvida por Dela Coleta e Dela Coleta (2006) e que busca determinar índices de felicidade e bem-estar e sua relação com o comportamento acadêmico. Foram utilizadas escalas sobre o sentimento de felicidade, satisfação, BES e aspectos da vida acadêmica. As escalas foram adaptadas daquelas apresentadas por Diener et al. (1995), Diener e Suh (2000) e Seligman (2004), com características psicométricas que recomendam sua utilização para a medida dessas variáveis. $\mathrm{O}$ conjunto dos instrumentos continha, ainda, uma questão aberta que solicitava aos sujeitos que fornecessem elementos definidores do que seria para eles uma vida boa.

Essas escalas utilizadas no trabalho foram adaptadas a partir da tradução para o português e de uma flexão semântica, mas não houve uma validação dessa bateria para o contexto brasileiro, o que abre a discussão acerca da utilização de tais instrumentos em nosso meio. Geralmente, as escalas não passam por uma adequada validação estatística, o que pode nos levar a considerar que a adaptação vem se mostrando útil, embora a sua valida- ção como instrumento passível de ser aplicado para a compreensão do contexto brasileiro ainda não possa ser atestada.

Dentre os instrumentos utilizados na pesquisa de Resende et al. (2006), o que se refere à mensuração do BES é a escala do tipo "item único" para medida da satisfação geral com a vida, referida por Neri (1998). É um instrumento para medida de BES indicado por satisfação geral com a vida, contendo duas questões que avaliam a satisfação com a vida atual e futura, por meio da figura de uma escada de dez degraus, que simboliza uma escala de dez pontos entre a pior vida e a melhor vida. São formuladas duas questões, uma referente à satisfação com a vida atual e outra, sobre a expectativa de satisfação com a vida nos próximos cinco anos.

Paschoal e Tamayo (2008) afirmam que, assim como as proposições teóricas acerca do bem-estar variam, os instrumentos para mensuração também são distintos. Resgatando as considerações de Diener (1984) sobre o bem-estar subjetivo, os autores afirmam que o afeto pode ser classificado em positivo e negativo e suas medidas não são opostas, mas apresentam apenas correlação negativa moderada e são claramente independentes. As dimensões positiva e negativa do afeto têm sido confirmadas em estudos conduzidos em diferentes culturas (Albuquerque \& Trócolli, 2004).

Resumidamente, de acordo com Paschoal e Tamayo (2008), as medidas de afeto positivo refletem o quanto a pessoa sente-se entusiasmada, alerta e ativa. Já o afeto negativo reflete o quanto a pessoa sente desprazer e distress. Segundo esses autores, uma das principais medidas de afeto utilizadas no estudo do bem-estar geral é a Positive Affect/Negative Afecct Scale ([PANAS], Watson, Clark, \& Tellegen, 1988), composta por 20 itens que mensuram o afeto negativo e o afeto positivo.

O estudo de Ferraz et al. (2007), em um de seus tópicos, revisa as escalas e os questionários para avaliação de felicidade e de bem-estar subjetivo disponíveis na literatura. É relevante mencionar que este foi o único artigo encontrado que se dedica às formas de mensuração da felicidade, ou do bem-estar e da satisfação, a partir da perspectiva da Psicologia Positiva.

Outro instrumento bastante utilizado, segundo Ferraz et al. (2007), é a PANAS-X ([Positive and Negative Affect Schedule - Expanded Form], Watson \& Clark, 1991), que é a versão expandida da PANAS. Esse questionário de autoavaliação é composto por 60 palavras, que descrevem sentimentos que devem ser respondidos de acordo com uma escala de cinco pontos: "muito pouco ou nada", "um pouco", "moderadamente", "muito" e "excessivamente", permitindo o uso como medida de estado ou de traço. A PANAS-X baseia-se em dois amplos fatores gerais: afeto positivo (jovialidade, autoconfiança, atenção, surpresa, serenidade) e afeto negativo (medo, tristeza, culpa, hostilidade, timidez, cansaço e surpresa) (Ferraz et al., 2007). 
Uma das questões que envolvem as medidas de afeto refere-se à influência indevida do humor momentâneo do indivíduo sobre suas respostas aos itens. Dos instrumentos abordados, segundo Ferraz et al. (2007), o mais frequentemente mencionado é a SWLS (Satisfaction with Life Scale), desenvolvida por Diener, Emmons, Larsen e Griffin (1985) e que verifica o julgamento pessoal que o indivíduo faz sobre sua qualidade de vida. Trata-se de um instrumento unidimensional de cinco itens com respostas entre um ("discordo fortemente") e sete ("concordo fortemente"), totalizando escore mínimo de cinco (menor satisfação) e máximo de 35 (maior satisfação). O índice de consistência interna é considerado bom (alfa de Cronbach $=0,87$ ) e a confiabilidade teste-reteste após dois meses é de 0,82, conforme Ferraz et al. (2007). Outro instrumento de destaque é o Oxford Happiness Inventory ([OHI], Argyle, Martin, \& Crossland, 1989), um questionário composto por 29 itens, que avalia as causas psicológicas gerais da felicidade, incluindo realização, satisfação, vigor e saúde. Sua confiabilidade teste-reteste é de 0,78 e o alfa de Cronbach é igual a 0,93 (Ferraz et al., 2007).

Há também a Life Satisfaction Inventory: Form A (Liang, 1984; Neugarten, Havinghurst, \& Tobin, 1961), que possui as seguintes categorias: entusiasmo pela vida, resolução e fortaleza, congruência entre os objetivos pretendidos e alcançados, fatores físicos, psicológicos, sociais e autoconceito.

A Subjective Happiness Scale ([SHS], Lyubomirsky \& Lepper, 1999) é um índice de felicidade subjetiva que compreende quatro itens. A média das respostas compõe um escore composto que varia de 1 a 7. A SHS apresenta consistência interna elevada (alfa de Cronbach entre 0,85 e 0,95 em diferentes estudos), estrutura unitária, boa confiabilidade teste-reteste (entre 0,71 e 0,90 ) e boa correlação com a avaliação de informantes $(r=0,65)$. Sua validade de constructo foi examinada em diversos estudos, apresentando boa correlação (entre 0,61 e 0,69) com a SWLS (Ferraz et al., 2007).

Ferraz et al. (2007) destacam, ainda, a DepressionHappiness Scale, um instrumento unidimensional de estrutura bipolar, em que um polo é representado pela felicidade e o outro, pela depressão. É composta por 25 itens e possui boa correlação com o Oxford Happiness Inventory. A sua versão abreviada, a Short DepressionHappiness Scale, é composta por seis itens e conserva boas propriedades psicométricas de consistência interna, confiabilidade teste-reteste e validade discriminante.

Como instrumento desenvolvido no Brasil, tem-se a Escala de Bem-estar Subjetivo ([EBES], Albuquerque \& Tróccoli, 2004). A EBES é um instrumento inspirado em escalas existentes no exterior (Escala de Afeto Positivo e Afeto Negativo - PANAS, de Watson et al., 1988; da Escala de Satisfação com a Vida - SWLS, de Diener et al., 1985; e Escala de Bem-estar Subjetivo - SWBS, de Lawrence \& Liang, 1988, citadas por Albuquerque \&
Tróccoli, 2004), com itens elaborados e analisados em grupos de validação semântica. Como muitos termos traduzidos não apresentavam clareza para os respondentes brasileiros, alguns itens foram adaptados para a cultura nacional e outros novos foram incluídos (Paschoal \& Tamayo, 2008).

A área do bem-estar subjetivo (BES) possui suas bases empíricas nas medidas de autorrelato de Diener (1984). A Escala de Bem-estar Subjetivo - EBES, de Albuquerque e Tróccoli (2004), é composta por três fatores: afeto positivo (21 itens e alfa de Cronbach de 0,95), afeto negativo (26 itens e alfa de Cronbach de 0,95) e satisfação com a vida (15 itens e alfa de Cronbach de $0,90)$. O instrumento é composto por duas subescalas de resposta tipo Likert de cinco pontos. Na primeira parte da escala, os itens vão do número 1 ao 47 e descrevem afetos positivos e negativos, devendo o sujeito responder como tem se sentido ultimamente em uma escala na qual um significa nem um pouco e cinco significa extremamente. Na segunda parte da escala, os itens variam do número 48 ao 62 e descrevem julgamentos relativos à avaliação de satisfação ou insatisfação com a vida, devendo ser respondidos em uma escala na qual um significa discordo plenamente e cinco significa concordo plenamente.

Como pôde ser constatado nesta revisão, há apenas um instrumento desenvolvido no âmbito nacional. Ainda assim, a EBES é uma escala inspirada em instrumentos internacionais como PANAS, SWLS e SWBS e foi validada para uma amostra específica, constituída por policiais militares do Distrito Federal. Os demais instrumentos mencionados são internacionais e, quando aplicados em pesquisas brasileiras, foram apenas traduzidos e/ou adaptados semanticamente, sem que um estudo de validação fosse empreendido dentro de rígidos parâmetros estatísticos. Não se encontrou qualquer registro de instrumento elaborado e validado no Brasil a partir de pesquisas nacionais como, por exemplo, a criação de uma escala de avaliação de aspectos contemplados pela Psicologia Positiva (bem-estar subjetivo, resiliência, otimismo, esperança, satisfação com a vida), a partir de estudo empírico que investigasse tais aspectos em uma determinada população.

Nesse sentido, recomenda-se que a EBES seja utilizada em mais pesquisas que comprovem sua adequação ao contexto brasileiro e que sejam desenvolvidas outras formas de avaliação que destaquem, fundamentalmente, os elementos e especificidades de nosso contexto. Isso se mostra relevante a fim de que a compreensão dos aspectos positivos e adaptativos possa se dar de modo situado e contextualizado, o que pode, inclusive, contribuir para que estudos transculturais elucidem as semelhanças e diferenças entre a percepção de felicidade e bem-estar subjetivo de diferentes culturas. Essa perspectiva intercultural não pode se reduzir apenas a pesquisar as possíveis diferenças entre os escores, mas aquilo que é abarcado 
pelas culturas quando avaliam subjetivamente esses aspectos da vida.

\section{Considerações Finais}

Concluindo o percurso desta revisão integrativa, assim como destacado no trabalho de Paludo e Koller (2007), o interesse pelos fenômenos positivos e aspectos saudáveis têm aumentado nos últimos anos, tanto no Brasil como no mundo. Essa perspectiva traduz uma mudança de olhar em relação ao humano, o que ocorre sempre que um novo campo de saber desponta. Apesar de recente, o movimento da Psicologia Positiva tem produzido importantes aplicações e avanços científicos. Constata-se a existência de uma tendência positiva devido ao crescente número de publicações no cenário científico internacional, o que evidencia o interesse dos pesquisadores nessas temáticas, que servem de inspiração para os autores nacionais.

No Brasil, Paludo e Koller (2007) revelam que o movimento da Psicologia Positiva ainda não recebeu a devida atenção, o que é corroborado pelos dados sistematizados na presente revisão. Apesar dessa relativa negligência por parte dos estudos nacionais, pode-se perceber que o número de trabalhos está aumentando, notadamente os de revisão, que se preocupam não apenas em apontar tendências, mas em resgatar o histórico desse movimento, a fim de entender seus pressupostos de base, seus avanços, limites e possibilidades. Esses trabalhos podem contribuir para a formulação de novos estudos empíricos nessa vertente, algo que foi destacado como uma lacuna na presente revisão.

Pensando ainda no contexto brasileiro e na produção científica dedicada à Psicologia Positiva, pode-se dizer que esse campo ainda se encontra em processo de desenvolvimento, mas com perspectiva promissora de consolidação no futuro. Embora ainda incipiente, a produção de novos conhecimentos permite antever um campo fecundo para a Psicologia brasileira. O número ainda reduzido de trabalhos pode ser atribuído ao caráter recente dos trabalhos produzidos na área, uma vez que essa corrente nasce, oficialmente, no final da década de 1990 e início dos anos 2000.

Um outro elemento permite aferir que a repercussão da Psicologia Positiva no cenário brasileiro ainda não é expressiva. Basta lançarmos um olhar para os congressos e eventos científicos no país. A Psicologia Positiva ainda não conquistou um espaço específico para a discussão de seus estudos, sendo que sua assunção e desenvolvimento vêm se dando na interface com outras áreas, notadamente com a Psicologia da Saúde (Calvetti et al., 2007).

Do ponto de vista da avaliação na Psicologia Positiva, relacionada aos aspectos de bem-estar subjetivo e noções correlatas, como flow, self, satisfação, locus de controle, dentre outros, deve-se afirmar a necessidade de produção de instrumentos adaptados e validados para o contexto brasileiro (Albuquerque \& Tróccoli, 2004; Paschoal \& Tamayo, 2008), de preferência construídos a partir de amostras nacionais, o que também não impede que esses estudos investiguem os clássicos instrumentos internacionais, seus pressupostos, sua adaptação e validação transcultural e sua aplicabilidade (Ferraz et al., 2007) em diversas situações, populações e contextos culturais, como é o caso de idosos, dos portadores de transtornos mentais e de comportamento.

A partir dos resultados obtidos, constatou-se a predominância de instrumentos, seja no contexto nacional como internacional, que avaliam, em alguma medida, os domínios do bem-estar subjetivo, ou seja, afetos positivos, afetos negativos e satisfação com a vida. A maioria das escalas mencionadas privilegia um ou todos esses aspectos. Mas seriam apenas esses os domínios envolvidos com o bem-estar e com a assunção de aspectos adaptativos preconizados pela Psicologia Positiva? Aqui se abre mais uma lacuna de conhecimento, que não apenas deve impulsionar novas investigações no meio brasileiro, como no contexto internacional.

Assim, a partir da revisão dos instrumentos de mensuração e de avaliação existentes na perspectiva da Psicologia Positiva, pôde-se não apenas conhecer quais escalas, inventários ou questionários utilizados, mas também quais os principais pressupostos teóricos que vêm orientando a construção e o desenvolvimento dessas ferramentas. Pelos resultados deste trabalho, pode-se concluir que os instrumentos existentes estão centrados no conceito de bem-estar subjetivo. Embora isso possa significar possível amadurecimento da compreensão do BES e a potencialidade do uso dessa noção em diferentes trabalhos, pode deflagrar uma certa limitação existente na própria Psicologia Positiva, uma vez que, ao referirse aos aspectos positivos e adaptativos do ser humano, ainda o dimensiona a partir de apenas um construto. Será que esse construto seria suficiente para a mudança de foco e de atuação almejada por essa perspectiva? Obviamente, pela amostra encontrada neste estudo, deve-se ter cautela ao apontar um direcionamento. No entanto, essa primeira busca pode levar a outros estudos, que desvelem novas possibilidades de mensuração dos aspectos apregoados pela Psicologia Positiva.

Quais as possibilidades de aplicação desses instrumentos e quais as suas repercussões para a prática psicológica nos cenários nacional e internacional são questões que permanecem em aberto, sem uma resposta conclusiva. Em que medida o estudo da felicidade e dos aspectos positivos do desenvolvimento pode favorecer o desenvolvimento de políticas públicas adequadas à população, não negligenciando as capacidades de cada indivíduo, nem limitando a intervenção a práticas remediadoras? Qual o alcance social da Psicologia Positiva e sua potencial aplicabilidade na busca de soluções para os problemas candentes que afligem a maior parte da população 
brasileira? Questões como essas merecem a consideração dos pesquisadores, mediante a condução de planos de investigação adequados.

Assim, cada vez mais há necessidade de desenvolvimento de novos estudos que investiguem a Psicologia Positiva em seu cerne constitutivo, a fim de que esse campo promissor possa não apenas sobreviver, mas contribuir para um repensar contínuo acerca do ser humano, promovendo mudanças e intervenções bem-sucedidas em diferentes contextos de atuação: clínicos, educacionais ou organizacionais. O essencial é que as pesquisas se abram efetivamente para a necessidade de incorporarem essa rotação de perspectiva, mudança que encontra na literatura científica não apenas ressonância, mas também possibilidades geradoras de novas reflexões e ideias férteis.

\section{Referências}

Albuquerque, A. S., \& Tróccoli, B. T. (2004). Desenvolvimento de uma escala de bem-estar subjetivo. Psicologia: Teoria e Pesquisa, 20(2), 153-164.

Argyle, M., Martin, M., \& Crossland, J. (1989). Happiness as a function of personality and social encounters. In J. Forgas \& J. Innes (Eds.), Recent advances in Social Psychology: An international perspective (pp. 189-203). Amsterdam, Netherlands: Elsevier.

Beyea, S. C., \& Nicoll, L. H. (1998). Writing in integrative review. AORN Journal, 67(4), 877-880.

Calvetti, P. U., Muller, M. C., \& Nunes, M. L. T. (2007). Psicologia da Saúde e Psicologia Positiva: Perspectivas e desafios. Psicologia: Ciência e Profissão, 27(4), 706-717.

Caprara, G. V., \& Steca, P. (2006). Affective and interpersonal self-regulatory efficacy beliefs as determinants of subjective well-being. In A. Delle Fave (Ed.), Dimensions of well-being: Research and intervention (pp. 120-142). Milan, Italy: FrancoAngeli.

Contreras, F., \& Esguerra, G. (2006). Psicología Positiva: una nueva perspectiva en psicologia. Diversitas: Perspectivas em Psicología, 2(2), 311-319.

Csikszentmihalyi, M. (2006). Introduction. In: M. Csikszentmihalyi \& I. S. Csikszentmihalyi (Eds.), A life worth living: Contributions to Positive Psychology (pp. 314). New York: Oxford University Press.

Dell'Aglio, D. D., Koller, S. H., \& Yunes, M. A. (2006). Resiliência e Psicologia Positiva: Interfaces do risco à proteção. São Paulo, SP: Casa do Psicólogo.

Dela Coleta, M. F., \& Dela Coleta, J. A. (2006). Felicidade, bem-estar subjetivo e comportamento acadêmico de estudantes universitários. Psicologia em Estudo, 11(3), 533-539.

Diener, E. (1984). Subjective well-being. Psychological Bulletin, 95, 542-575.

Diener, E., Diener, M., \& Diener, C. (1995). Factors predicting the subjective well being of nations. Journal of Personality and Social Psychology, 69, 851-864.

Diener, E., Emmons, R. A., Larsen, R. J., \& Griffin, S. (1985). The satisfaction with Life Scale. Journal of Personality Assessment, 49, 71-75.

Diener, E., \& Suh, E. M. (2000). Culture and subjective wellbeing. Cambridge, MA: MIT Press.
Ferraz, R. B., Tavares, H., \& Zilberman, M. L. (2007). Felicidade: Uma revisão. Revista de Psiquiatria Clínica, 34(5), 234-242.

Graziano, L. D. (2005). A felicidade revisitada: Um estudo sobre bem-estar subjetivo na visão da Psicologia Positiva. Tese de Doutorado não-publicada, Instituto de Psicologia, Universidade de São Paulo, SP.

Ganong, L. H. (1987). Integrative reviews of nursing research. Research in Nursing \& Health, 10, 1-11.

Delle Fave, A. (Ed.). (2006). Dimensions of well-being. Research and intervention. Milan, Italy: Franco Angeli.

Guedea, M. T. D., Albuquerque, J. B., Tróccoli, B. T., Noriega, J. A. V., Seabra, M. A. B., \& Guedea, R. L. D. (2006). Relação do bem-estar subjetivo, estratégias de enfrentamento e apoio social em idosos. Psicologia: Reflexão e Crítica 19(2), 301-308.

Larrauri, B. G. (2006). Programa para mejorar el sentido del humor: porque la vida con buen humor merece la pena! Madrid, España: Pirámide.

Lawrence, R. H., \& Liang, J. (1988). Structural integration of the Affect Balance Scale and the Life Satisfaction Index A: Race, sex, and age differences. Psychology and Aging, 3, 375-384.

Liang, J. (1984). Dimensions of the life satisfaction index A: A structural formulation. Journal of Gerontology, 39, 613-622.

Lyubomirsky, S., \& Lepper, H. S. (1999). A measure of subjective happiness: Preliminary reliability and construct validation. Social Indicators Research, 46, 137-155.

Marujo, H. A., Neto, L. M., Caetano, A., \& Rivero, C. (2007). Revolução positiva: Psicologia Positiva e práticas apreciativas em contextos organizacionais. Comportamento Organizacional e Gestão, 13(1), 115-136.

Neri, A. L. (1998). Escala para avaliação de satisfação na vida referenciada a domínios. Manuscrito não-publicado, Universidade Estadual de Campinas, SP.

Neri, A. L. (2002). Bienestar subjetivo en la vida adulta y en la vejez: hacia una Psicologia Positiva en America Latina Revista Latino-americana de Psicologia, 34(1/2), 55-74.

Neugarten, B. L., Havinghurst, R. J., \& Tobin, S. S. (1961). The measurement of life satisfaction. Journal of Gerontology, 16, 134-143.

Paludo, S. S., \& Koller, S. H. (2007). Psicologia Positiva: Uma nova abordagem para antigas questões. Paidéia, 17(36), 9-20.

Park, N., \& Peterson, C. (2007). Methodological issues in Positive Psychology and the assessment of character strengths. In A. D. Ong \& M. H. M. van Dulmen (Eds.), Oxford Handbook of methods in Positive Psychology (pp. 292-305). New York: Oxford University Press.

Paschoal, T., \& Tamayo, A. (2008). Construção e validação da Escala de Bem-estar no Trabalho. Avaliação Psicológica, 7(1), 11-22.

Passareli, P. M., \& Silva, J. A. (2007). Psicologia Positiva e o estudo do bem-estar subjetivo. Estudos de Psicologia (Campinas), 24(4), 513-517.

Pereira, C. A. A. (1997). Um panorama histórico-conceitual acerca das subdimensões de qualidade de vida e do bemestar subjetivo. Arquivos Brasileiros de Psicologia, 49(4), 32-48.

Resende, M. C., Bones, V. M., \& Souza, I. S. (2006). Rede de relações sociais e satisfação com a vida de adultos e idosos. Psicologia para América Latina, 5. Retrieved December 15 , 2008, from http://www.psicolatina.org/Cinco/rede.html 
Rijavec, M., Brdar, I., \& Miljkovic, D. (2006). Extrinsic vs. intrinsic life goals, psychological needs and life satisfaction. In A. Delle Fave (Ed.), Dimensions of well-being: Research and intervention (pp. 91-105). Milan, Italy: FrancoAngeli.

Scorsolini-Comin, F., \& Amorim, K. S. (2008). Corporeidade: Uma revisão crítica da literatura científica. Psicologia em Revista, 14(1), 189-214.

Scorsolini-Comin, F., \& Santos, M. A. (2009). A Psicologia Positiva no contexto brasileiro: História, presente e perspectivas futuras. In S. N. Jesus, I. Leal, \& M. Rezende (Eds.), Experiências e intervenções em Psicologia da Saúde (pp. 862-878). Faro, Portugal: Universidade do Algarve.

Scorsolini-Comin, F., \& Santos, M. A. (2010). Avaliação do bem-estar subjetivo (BES). Aspectos conceituais e metodológicos. Interamerican Journal of Psychology, 44(3), 438-444.

Seligman, M. E. (2000). Positive Psychology: An introduction. American Psychologist Association, 55(1), 5-14.

Seligman, M. E. (2004). Felicidade autêntica: Usando a nova Psicologia Positiva para a realização permanente. Rio de Janeiro, RJ: Objetiva.

Seligman, M. E. P., Park, A. C., \& Steen, T., \& Peterson, C. (2006). A balanced psychology and a full life. In F. A. Huppert, N. Baylis, \& B. Keverne (Eds.), The science of well-being ( $2^{\text {nd }}$ ed., pp. 285-304). Oxford, UK: Oxford University Press.

Serbena, C. A., \& Raffaelli, R. (2003). Psicologia como disciplina científica e discurso sobre a alma: Problemas epistemológicos e ideológicos. Psicologia em Estudo, 8(1), 31-37.

Sheldon, K. M., \& King, L. (2001). Why Positive Psychology is necessary. American Psychologist, 56, 216-217.

Watson, D., \& Clark, L. A. (1991). Preliminary manual for the PANAS-X: Positive and negative affect scheduleexpanded form. Dallas, TX: Southern Methodist University.

Watson, D., Clark, L.A., \& Tellegen, A. (1988). Development and validation of brief measures of positive and negative affect: The PANAS scales. Journal of Personality and Social Psychology, 54, 1063-1070.

Yunes, M. A. M. (2003). Psicologia Positiva e resiliência: O foco no indivíduo e na família [Special issue]. Psicologia em Estudo, 8, 75-84. 